\title{
Curando dentro, cuidando fuera: mujeres latinoamericanas y prácticas terapéuticas en el contexto migratorio transnacional
}

| ${ }^{1}$ Muriel Lamarque, ${ }^{2}$ Lourdes Moro Gutiérrez |

Resumen: El siguiente artículo intenta reflexionar acerca de la relación que se construye entre las mujeres latinas migrantes y las prácticas terapéuticas, atendiendo a los roles designados o asumidos por ellas en los procesos de salud/enfermedad/atención. Se realizó una investigación etnográfica con mujeres caribeńas establecidas en Espańa, con el fin de indagar acerca de determinadas actividades curativas y de cuidado ejercidas por ellas, tanto al interior del hogar como hacia afuera. Además del componente descriptivo, se trabajó con las narrativas y significados específicos elaborados en torno a dichas ocupaciones. Su análisis permitió identificar facetas contradictorias y conflictivas en el desarrollo de la identidad y la experiencia migratoria femenina. Por un lado, las actividades de cuidado como principal alternativa laboral evidencian la desigualdad de oportunidades y ámbitos de incidencia social accesibles a las latinoamericanas. Al mismo tiempo reflejan mecanismos de reproducción de esquemas esencialistas, que naturalizan la división del trabajo en función de "cualidades" étnicas y de género. Por otro lado, el manejo de conocimientos específicos sobre curación, tratamiento y asistencia supone para las mujeres la posesión de un papel distintivo y potencialmente empoderador al interior de la comunidad como continuadoras de determinados saberes tradicionales.

> Palabras clave: migración; medicina popular; prácticas terapéuticas; cuidados; roles de género.

\author{
1 Psicología Social y Antropología, \\ Universidad de Salamanca. \\ Salamanca, España (muriel. \\ lamarque@gmail.com). \\ ORCID: 0000-0002-4149-2334 \\ 2 Psicología Social y \\ Antropología, Universidad de \\ Salamanca. Salamanca, España \\ (moro@usal.es). \\ ORCID: 0000-0003-2009-0413
}

Recebido em: 19/04/2019 Aprovado em: 29/10/2019 Revisado em: 02/06/2020 


\section{Introducción}

El colectivo inmigrante proveniente de América Latina constituye uno de los más numerosos de Espańa y, al igual que en otras partes del mundo, se encuentra en creciente proceso de feminización desde hace varios años (CASTLES; MILLER, 1998; COLECTIVO IOÉ, 2003; MARTÍNEZ-BUJÁN, 2011; UNDA; ALVARADO, 2012; OSO, 2018). El reconocimiento en las últimas décadas de las mujeres como posibles actoras en los procesos de desplazamiento, al igual que su visibilización dentro de la población activa, han orientado numerosas investigaciones sociales en materia de migraciones y género (AUBARELL, 2000).

El contacto intercultural, el establecimiento de determinadas políticas migratorias, junto con las transformaciones en el mercado laboral - entre otras cosas - han intervenido en la conformación de un contexto particular en la sociedad de destino. Si reconocemos que una gran proporción de las personas movilizadas transnacionalmente son mujeres, cabe analizar y preguntarse acerca de los lugares que ellas están ocupando en dicha sociedad, y las formas en que esto se articula con las estructuras en las que estaban inmersas previamente. De igual modo, resulta pertinente conocer el universo de posibilidades disponible para ellas y cómo son las estrategias de afrontamiento desplegadas ante esa realidad.

\section{Mujeres pacientes, mujeres cuidadoras, mujeres en la atención}

Un análisis delos antecedentes específicos en materia de salud y migración femenina latinoamericana nos ha permitido identificar, hasta el momento, tres grandes ejes o tendencias temáticas. En primer lugar — relativos a enfoques de tipo demográfico, epidemiológico y sociológico - se agrupan aquellos trabajos acerca del uso de los servicios públicos de salud, los principales motivos de consulta o las problemáticas culturales derivadas del contacto entre el personal sanitario y las mujeres extranjeras (RÍO-BENITO et al., 2003; REGIDOR et al., 2009; LLOSADA-GISTAU et al., 2012). Estas indagaciones, muy importantes para la evaluación institucional y para el registro del comportamiento colectivo, se han basado mayoritariamente en la perspectiva de la sociedad de destino, recopilando testimonios de los profesionales biomédicos o haciendo uso de estadísticas comparativas (GUIJÓN-SÁNCHEZ; JIMÉNEZ-RODRIGO; MARTÍNEZ-MORANTE, 2006). 
Seguido se sitúan las investigaciones respecto de la inserción migrante en el mercado internacional de servicio doméstico y de cuidados a terceros. Tales contribuciones refuerzan la discusión acerca de la feminización/etnización de las labores de cuidado, profundizando sobre el emplazamiento social y cultural de las mujeres en relaciones desiguales y de explotación (MARTÍNEZ-BUJÁN, 2010; HONDAGNEU-SOTELO; ESTRADA; RAMÍREZ, 2011; COMAS D' ARGEMIR, 2015; GREGORIO-GIL, 2017). Su mayor trascendencia ha sido en las áreas de la economía laboral, economía política y feminismo, vinculando la división del trabajo reproductivo con el modelo global capitalista. A pesar del creciente desarrollo de estos temas, aún son escasas las producciones que analicen el ejercicio femenino de cuidado en términos de una práctica sanitaria invisibilizada, estimando su posible impacto a nivel comunitario y subjetivo.

Por último se encuentran aquellos estudios que han identificado a las migrantes latinoa mericanas como sustanciales proveedoras de salud familiar y grupal. Estos textos recuperan desde costumbres curativas populares o tradicionales (MENDELSON, 2003; WALDSTEIN, 2010; MORGAN-CONSOLI; UNZUETA, 2018; BAEZA; AIZENBERG; BARRÍA, 2019) hasta redes locales y trasnacionales de información y atención (MESSIAS, 2002; MENJÍVAR, 2002; SÁNCHEZ-BIRKHEAD et al., 2011). Lejos de ser pensados desde una lógica mercantil, en este caso los cuidados y la asistencia forman parte de estructuras de reciprocidad y cooperación, de amplio significado cultural.

En líneas generales, podemos decir que en el tratamiento académico de la experiencia migrante femenina ha prevalecido un abordaje fragmentado, restringido a ciertas esferas de la vida como la institucional, la laboral, la familiar, etc. Este artículo pretende contribuir al conocimiento preexistente mediante un análisis compuesto, que recorra el "tránsito" de estas mujeres por distintas formas de cuidado, en tanto prácticas de evidente valor sanitario. La mayoría de estas actividades son desarrolladas de manera informal —es decir, por fuera de los contextos institucionales o profesionales - y cuentan con escaso reconocimiento social. Nos hemos propuesto indagar acerca de tales acciones y, por lo tanto, profundizar en la relación que se establece entre las mujeres y las actividades terapéuticas, tomando como ejemplo el caso de las migrantes caribeñas establecidas en la región española de Castilla y León. Desde un enfoque antropológico se analizaron las prácticas 
y saberes específicos en materia de salud, ejercidos por dicho colectivo a lo largo del espacio migratorio. En particular se examinaron las tradiciones de curación doméstica y medicina popular empleadas al interior de la familia y comunidad, así como también las tareas remuneradas de responsabilización y cuidado de terceros, igualmente importantes en la conformación de entornos saludables (MENDELSON, 2003; DYCK; DOSSA, 2007).

A pesar de que existen claras diferencias entre estas actividades, hemos elegido conjugarlas aquí porque es posible identificar varios puntos de contacto o características comunes. En primer lugar, las dos se vinculan directamente con el mantenimiento, la vigilancia o la restauración de la salud. Considerando la perspectiva biopsicosocial, la salud es producto de una interacción entre dimensiones contextuales, físicas, emocionales, espirituales y del entorno vincular (JUÁREZ, 2011). De ello se desprende que las acciones que incidan sobre cualquiera de estos elementos puedan afectar positiva o negativamente sobre los niveles de salud/ enfermedad. En segundo lugar, dichas tareas son normalmente ejercidas en el ámbito privado, ya sea familiar personal o el espacio doméstico de terceros. Por último - y esta probablemente sea la característica más significativa — el ejercicio de la atención terapéutica informal y del cuidado están frecuentemente asociados al género femenino, y por ende, acompañados de discursos que justifican y naturalizan esta división genérica en los roles.

Durante el desarrollo de esta investigación intentamos recuperar los discursos, descripciones y explicaciones elaboradas por las propias mujeres involucradas; con el fin de comprender lo que el ejercicio de las prácticas curativas significa para ellas en términos de conformación de su identidad, y en tanto forma de vinculación con el nuevo territorio de vida.

\section{Metodología}

Este trabajo forma parte de una investigación antropológica más amplia acerca de las prácticas terapéuticas y estrategias de atención en migrantes latinoamericanos en España. Se realizó trabajo de campo etnográfico en la Comunidad Autónoma de Castilla y León entre los meses de noviembre de 2016 a julio de 2018. Se seleccionaron como informantes 18 mujeres de entre 33 y 71 años, provenientes de países del Gran Caribe (específicamente Colombia, Venezuela, Honduras, Guatemala, México, 
República Dominicana y El Salvador) y que tuvieran al menos un año de residencia en España. Este recorte geográfico se sustenta en que, a pesar de reconocer la existencia de variabilidad y características locales, consideramos que esta región presenta elementos culturales y sociohistóricos comunes o semejantes, que pueden contribuir en la identificación de experiencias análogas entre sus representantes.

Además de los relatos proporcionados por cada una de las participantes, se registraron datos sociodemográficos específicos, como el estado civil, la estructura familiar, el nivel educativo, la ocupación y la condición administrativa. Estos datos facilitaron la caracterización y clasificación de las informantes, así como también detalles contextuales pertinentes en materia de salud, como la red social próxima, la tenencia de empleo registrado y la posibilidad de acceso a atención sanitaria pública. Los mismos son detallados en la tabla 1 .

Las técnicas de recolección de datos fueron la observación participante y la realización de entrevistas en profundidad. Se confeccionó un guion temático orientativo de preguntas, basado en las siguientes categorías: a) Características del proceso migratorio personal, b) Creencias y significados respecto de la salud y la enfermedad, c) Actividades y prácticas para el cuidado de la salud y el tratamiento de la enfermedad, d) Elecciones, causas y fuentes y, e) Roles al interior del grupo vincular. Los contactos iniciales con algunas de las informantes se realizaron en asociaciones locales de asistencia a población migrante, compartiendo con ellas distintas actividades recreativas organizadas allí. Otras personas fueron seleccionadas mediante la técnica de bola de nieve, por sugerencia o recomendación de las mujeres colaboradoras. Las actividades de observación se realizaron además en unidades domésticas, tiendas comerciales e instituciones de asistencia sanitaria, acompañando a distintos sujetos en actividades relativas al cuidado o atención de la enfermedad.

Tras explicar y detallar los propósitos y características del estudio se procedió a registrar el consentimiento expreso de cada una, al mismo tiempo que se les solicitó permiso para grabar en audio digital el contenido de las entrevistas. La conducta ética de este estudio se adscribe al código deontológico para la práctica profesional de la antropología, establecido por la Asociación Antropológica del Estado Español (ASSAE, 2014).

Las entrevistas se transcribieron textualmente, y se seleccionaron algunos fragmentos para ilustrar este documento. A fin de preservar la identidad de todos los sujetos involucrados, los nombres presentados junto a los extractos testimoniales son ficticios. 
Por último, la información obtenida en el trabajo de campo se procesó mediante análisis temático (GUEST; MACQUEEN; NAMEY, 2012; MIELES-BARRERA; TONON; ALVARADO-SALGADO, 2012) creando códigos o categorías sistemáticos basados principalmente en el discurso y complementados con la observación. Inspeccionar las narrativas de las mujeres sobre diversos aspectos de su historia y cotidianeidad permitió —además de acceder a la descripción de las prácticas culturalmente específicas - explorar los significados, las interpretaciones y los valores relacionados con la atención de la salud (KLEINMAN, 1988; HAMUI, 2011).

\section{Resultados y Discusión}

\section{Las formas de la tradición: atención y curación femenina al interior del hogar}

En múltiples contextos culturales latinoamericanos, es habitual que ante un padecimiento o malestar sean las mujeres las encargadas de iniciar la resolución de los problemas respecto del proceso salud/enfermedad/atención (MENÉNDEZ, 1993; OSORIO-CARRANZA, 2001, 2007; BARTOLI, 2005; WALDSTEIN, 2010). Generalmente estas actividades se desempeñan en el ámbito del hogar e incluyen acciones como el monitoreo de las funciones corporales de los miembros de la familia, la traducción de signos a síntomas y la construcción de explicaciones y significados que conduzcan a un diagnóstico inicial (PEZO-SILVA; DE SOUZA-PRAÇA; COSTASTEFANELLI, 2004). Tras estas valoraciones, se procede a elegir el tratamiento más adecuado, de acuerdo con eficacias y experiencias anteriores, creencias y posibilidades, entre otros factores (KLEINMAN, 1978; MEDINA-CÁRDENAS, 1998).

Las mujeres entrevistadas durante la investigación señalaron ocuparse personalmente de gran parte de las dolencias emergentes en el seno de su familia, desplegando una serie de recursos domésticos comunes para el tratamiento de niños y adultos.

Mi marido trabajaba con cemento, y tenía muy malos los pulmones. Siempre tos, siempre flema. Yo le empecé a dar berro con leche y miel tres veces al día. Y con eso logré que botara toda la flema y limpiara los pulmones en una semana (Judith, 42 ańos, Venezuela). 


\begin{tabular}{|c|c|c|c|c|c|c|c|c|c|c|c|c|c|c|c|c|c|c|}
\hline 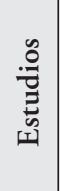 & 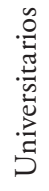 & 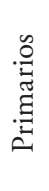 & 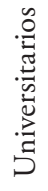 & 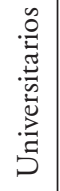 & 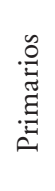 & 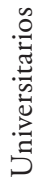 & 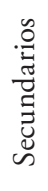 & 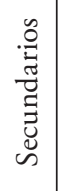 & 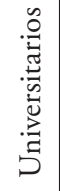 & 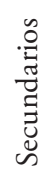 & 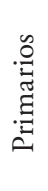 & 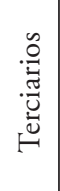 & 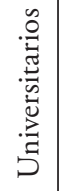 & 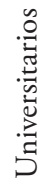 & 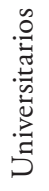 & 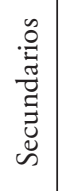 & 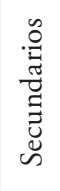 & 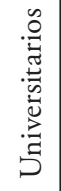 \\
\hline$\frac{8}{\frac{\Xi}{\tilde{z}}}$ & 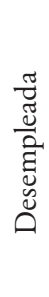 & 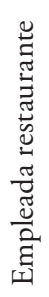 & 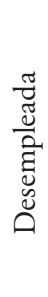 & 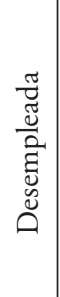 & 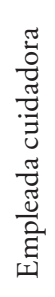 & 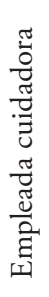 & 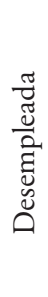 & 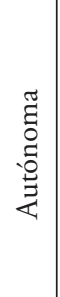 & 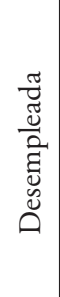 & 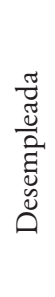 & 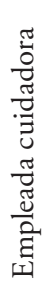 & $\begin{array}{l}\frac{\tilde{J}}{\tilde{J}} \\
\frac{\tilde{D}}{0} \\
\tilde{\Xi} \\
\tilde{D} \\
0\end{array}$ & 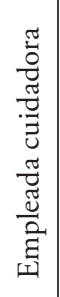 & 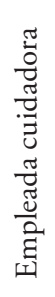 & 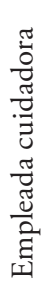 & $\begin{array}{l}\frac{\tilde{J}}{\tilde{J}} \\
\frac{\tilde{D}}{0} \\
\tilde{\Xi} \\
\tilde{\Xi} \\
\tilde{0}\end{array}$ & 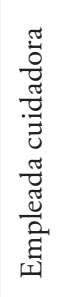 & 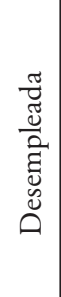 \\
\hline 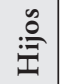 & $i s$ & $\ddot{n}$ & $\stackrel{\circ}{Z}$ & $\dot{Z}$ & $i n$ & $\ddot{n}$ & $i$ & is & is & $i n$ & $\stackrel{\circ}{Z}$ & $i n$ & $i \vec{s}$ & in & 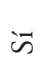 & $\ddot{\omega}$ & is & $\stackrel{\circ}{z}$ \\
\hline 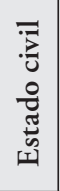 & $\begin{array}{l}\underset{\widetilde{J}}{\widetilde{J}} \\
\tilde{\widetilde{U}} \\
\tilde{U}\end{array}$ & 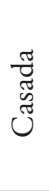 & 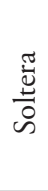 & $\begin{array}{l}\frac{\pi}{\pi} \\
\frac{\pi}{\pi} \\
\tilde{\pi}\end{array}$ & $\frac{\pi}{\tilde{U}}$ & $\begin{array}{l}\frac{\pi}{\pi} \\
\frac{\pi}{\pi} \\
\end{array}$ & $\stackrel{\widetilde{J}}{\Xi}$ & $\begin{array}{c}\frac{0}{\tilde{U}} \\
\tilde{J} \\
\tilde{U}\end{array}$ & $\begin{array}{l}\frac{\tilde{J}}{\widetilde{J}} \\
\tilde{\widetilde{U}} \\
\tilde{U}\end{array}$ & $\begin{array}{l}\widetilde{J} \\
\widetilde{\widetilde{J}} \\
\tilde{J}\end{array}$ & $\stackrel{\widetilde{0}}{\Xi}$ & $\begin{array}{l}\frac{\pi}{\tilde{E}} \\
\frac{D}{0}\end{array}$ & $\begin{array}{l}\underset{\tilde{J}}{\tilde{J}} \\
\tilde{\widetilde{U}} \\
\tilde{U}\end{array}$ & $\begin{array}{l}\widetilde{\widetilde{J}} \\
\text { J్ } \\
\tilde{J}\end{array}$ & 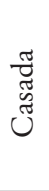 & $\begin{array}{l}\frac{\pi}{\tilde{U}} \\
\frac{\tilde{D}}{0}\end{array}$ & 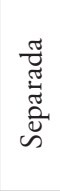 & $\begin{array}{l}\frac{\pi}{\pi} \\
\stackrel{\pi}{\pi} \\
\tilde{a}\end{array}$ \\
\hline 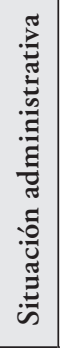 & 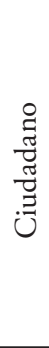 & 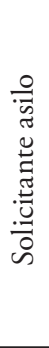 & 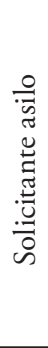 & 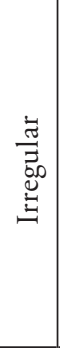 & 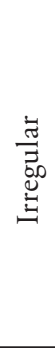 & 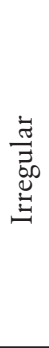 & 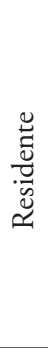 & $\begin{array}{c}\stackrel{\tilde{U}}{0} \\
\frac{0}{0} \\
\tilde{u} \\
\tilde{c}\end{array}$ & 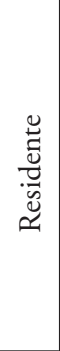 & 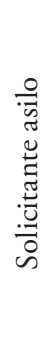 & 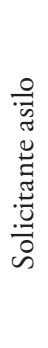 & 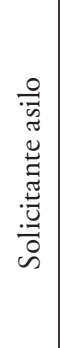 & 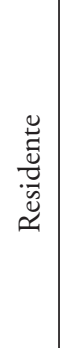 & 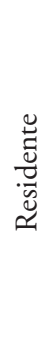 & 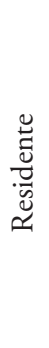 & 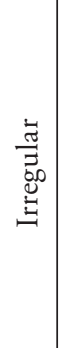 & 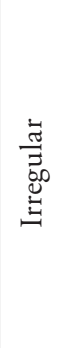 & 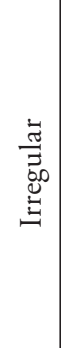 \\
\hline$\underset{\tilde{T}}{\vec{T}}$ & $\mathscr{F}$ & 아 & $\bar{n}$ & 아 & 요 & $\stackrel{\circ}{n}$ & $\nabla$ & $F$ & $\tilde{m}$ & F & $\vec{\forall}$ & $\tilde{n}$ & 아 & † & $\tilde{n}$ & mे & ते & ৩ \\
\hline$\stackrel{\frac{n}{\pi}}{=}$ & 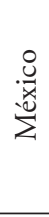 & 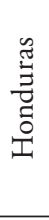 & $\begin{array}{l}\frac{\pi}{\mathbb{U}} \\
\mathbb{N} \\
\tilde{U} \\
> \\
\end{array}$ & $\begin{array}{l}\frac{\pi}{0} \\
\frac{\tilde{E}}{0} \\
0 \\
0\end{array}$ & 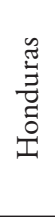 & $\begin{array}{l}\frac{\pi}{\Delta} \\
\stackrel{N}{N} \\
\stackrel{0}{0} \\
>\end{array}$ & 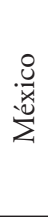 & $\begin{array}{c}\frac{\tilde{J}}{\tilde{J}} \\
\tilde{\Xi} \\
\tilde{J} \\
\tilde{J} \\
\tilde{J}\end{array}$ & 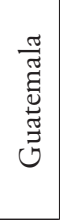 & 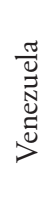 & 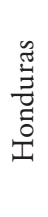 & 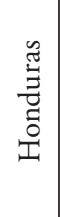 & 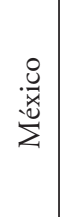 & 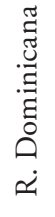 & 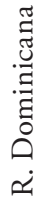 & 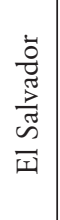 & $\begin{array}{l}\underset{\widetilde{J}}{\Xi} \\
\stackrel{\Xi}{\Xi} \\
\stackrel{0}{I}\end{array}$ & 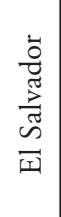 \\
\hline ت્ & 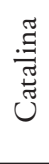 & $\frac{\widetilde{g}}{\stackrel{\Xi}{0}}$ & 营 & $\frac{\widetilde{m}}{\frac{0}{a}}$ & 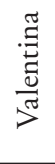 & 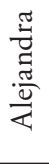 & 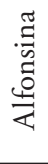 & $\stackrel{\frac{\pi}{U}}{\underset{Z}{*}}$ & $\begin{array}{l}\stackrel{\pi}{3} \\
\stackrel{w}{5}\end{array}$ & 吾 & 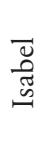 & 㞼 & 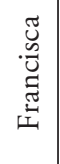 & 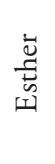 & 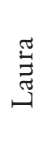 & 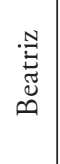 & $\stackrel{\widetilde{Z}}{\mathscr{Z}}$ & 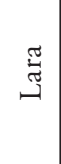 \\
\hline & - & $\sim$ & $n$ & $*$ & in & 6 & $\Lambda$ & $\infty$ & $a$ & $\stackrel{ }{ }$ & $\exists$ & $\mathcal{\simeq}$ & 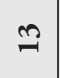 & $\Xi$ & $\cong$ & ? & $\cong$ & $\stackrel{\infty}{\sim}$ \\
\hline
\end{tabular}


En casi la totalidad de los casos, los conocimientos de medicina casera fueron adquiridos en los países de origen, mediante observación o trasmisión oral, generalmente por vía lineal materna. Son especialmente las figuras femeninas mayores — madres, tías, abuelas — quienes transmiten lo acumulado por la historia y la experiencia, en el transcurso mismo de la vida cotidiana (WILKINSON, 1987; JORAND, 2008, SÁNCHEZ-BIRKHEAD et al., 2011):

Todo eso imagínate que viene desde mi abuela, por mi mamá (Valentina, 50 años, Honduras).

Mi mami me daba esas medicinas. Siempre son las mujeres las que se ocupan de esas cosas. Desde la primera vez que me vino la menstruación mi mami me daba (Lydia, 35 años, Honduras).

Esas recetas me las pasa mi madre, o mi abuela. Ellas son las fuentes de consulta» (Lara, 36 años, El Salvador).

Las mujeres señalaron confiar en la experiencia de las generaciones anteriores para proveer los cuidados y atenciones necesarias en materia de salud familiar. La eficacia de estas acciones terapéuticas adquiere entonces un carácter histórico, que se comprueba periódicamente al recuperar las fórmulas aprendidas. Sus relatos sacan a relucir, entonces, el factor o la dimensión social de las prácticas femeninas, al evocar la asistencia colectiva y la transferencia de saberes dentro de un mismo círculo de mujeres. Así lo cuenta, por ejemplo, Alfonsina, quien recuerda la intervención de sus parientes aconsejando a una de sus hijas, quien iba a dar a luz luego de migrar:

Yo me acuerdo de cuando iba a nacer su primera hija aquí, pues trajo de México todas sus hierbas. Porque después del parto se lo dijeron todas en la familia: "que te hagas un baño con estas hierbas". Para limpiar las heridas que quedan [...] Las mujeres que ya han pasado por eso te dicen cómo... (Alfonsina, 71 años, México)

Los testimonios con frecuencia evocaron e insistieron en la utilización de este tipo de recetas curativas como la continuación de un legado femenino "tradicional":

Todo eso lo aprende una en casa, que le decían "si tienes, por ejemplo, cólicos menstruales, entonces toma manzanilla con ortiga" Y bueno... Continuamos con lo que las abuelas han dejado... (Eloísa, 40 años, Colombia).

En este sentido, la socialización de las mujeres en el marco familiar/hogareño conlleva una clara distribución de roles, estableciendo para ellas el cuidado de los miembros del grupo (SILES-GONZALEZ, SOLANO-RUIZ, 2007; VAQUIRORODRÍGUEZ, STIEPOVICH-BERTONI, 2010; ZAPPAROLI-ZECCA, 2012). Los significados que allí se establecen son naturalizados y perpetuados en 
los procesos de trasmisión cultural, enseñando a las generaciones siguientes acerca de la estructura de los roles y sus rasgos de comportamiento (ORTNER, 1979). Atender a hijos, padres, nietos y parejas suele ser planteado en términos de una responsabilidad cultural (MENDELSON, 2003), incorporándose como parte de las expectativas de comportamiento social (HERRERA-SANTI, 2000). Como recoge Bermúdez-Quintana (2006) en su propio trabajo con mujeres colombianas, salirse del patrón de cuidado y responsabilización sobre los otros implica un reproche social, mientras que cumplir con el mandato es reafirmado con la idea de "buena mujer". En la comunidad latina radicada en España pudo constatarse esta división de tareas. El dominio de las prácticas curativas y asistenciales se adiciona al resto de las cargas domésticas destinadas comúnmente a las mujeres. Con este señalamiento no pretendemos negar ni invisibilizar la existencia de figuras masculinas en el ejercicio terapéutico intrafamiliar, sino que resaltamos la mayoritaria recurrencia de la ocupación femenina en el mismo.

El empleo de estos recursos curativos fue sostenido en forma continuada por todas las mujeres entrevistadas, a pesar de las relocalizaciones del campo a la ciudad, o entre países. Al igual que Velasco (2002), consideramos el espacio migratorio como un continuo social, que incluye simultáneamente a los territorios de origen y destino. En el caso de las participantes, el mantenimiento del legado terapéutico tradicional fue revestido de un valor positivo como "puente" cultural, al mismo tiempo que implica ciertas formas de reivindicación identitaria, mediante la adscripción y sostenimiento de determinados principios familiares y colectivos como la solidaridad y la autosuficiencia:

Pero tenemos en nuestras venas esa cultura, de curarnos con los productos de la naturaleza. Y lo aprendemos y se lo explicamos a nuestros hijos, entonces se pasa de generación en generación (Esther, 47 años, República Dominicana).

No importa dónde vamos, siempre nos curamos así. Es la tradición... (Judith, 42 años, Venezuela).

Me gusta haber aprendido estas cosas naturales y ayudar a otros. Así somos nosotros los latinos, y es bueno que los de aquí lo puedan ver (Isabel, 41 años, Honduras).

Su conservación puede contribuir, asimismo, en el desarrollo de estrategias y sentimientos de resiliencia, al reconocer que se cuenta con recursos para enfrentar posibles adversidades (GENTIL, 2009; MORGAN-CONSOLI; UNZUETA, 2018). 
No obstante, también pudo evidenciarse el carácter dinámico de las terapias domésticas, dando lugar a nuevas formas de atención, nuevos saberes y transformaciones (HERNÁNDEZ-TEZOQUIPA; ARENAS-MONREAL; VALDE-SANTIAGO, 2001; VOLPATO; GODÍNEZ; BEYRA, 2009). A nivel comunitario se describieron intercambios de información con personas cercanas, especialmente otras mujeres migrantes que habitan el mismo barrio o con quienes comparten actividades:

Cuando hablas en la comunidad con otras latinas, o también las turcas, ellas también son mucho de hierbas, alternativas. Ellas me decían "mastica menta... té de canela" (Catalina, 45 años, México).

Ahora hemos conocido en el curso de cocina a una muchacha que es colombiana y ella sabe bastante de esas cosas. En una emergencia, al no poder ubicarla a mi mamá, pues yo creo que le preguntaría a ella. Gente así es bueno conocer porque uno aprende de ellos» (Rita, 39 años, Honduras).

Compartir y recibir recetas con otras migrantes parece ayudar a estas mujeres a ampliar el acervo personal, pero lo que es más importante, contribuye en el establecimiento de vínculos interétnicos de solidaridad, fortalecidos por el sentimiento de una experiencia común.

En términos generales, el tipo de remedio casero más referenciado y consumido fueron los preparados fitoterapéuticos. Su uso se encuentra ampliamente extendido en la medicina doméstica latinoamericana y ha formado parte de los hábitos curativos durante generaciones (ORGANIZACIÓN PANAMERICANA DE LA SALUD, 1999; BARRAGÁN-SOLÍS, 2006; IDOYAGA-MOLINA, 2007). La mayor parte de las informantes mencionó el empleo frecuente de plantas medicinales —en ocasiones combinadas con productos de origen animal o mineral - para tratar numerosas enfermedades y malestares de diversa etiología y gravedad tanto en niños como en adultos. Se documentaron recetas para dolores de cabeza, problemas estomacales, resfriados y catarros, dolores de oídos, insomnio y problemas nerviosos. De igual modo se mencionó el consumo preventivo de preparados para aumentar las defensas, bajar de peso y mejorar la digestión. Como una parte especial de los saberes médicos autoadministrados, las participantes destacaron también el manejo de cuidados específicos respecto de enfermedades ginecológicas, tratamientos de fertilidad y atenciones durante embarazo y lactancia: 


\begin{abstract}
Por ejemplo, cuando tú tienes alguna infección en la vagina, bueno, nosotros usamos el romero. Lo hervimos, lo colamos, lo echamos en una bombita y la metemos adentro. Te lo vas haciendo cada, más o menos, seis meses (Alicia, 41 años, Guatemala).

El maíz te sirve para mil cosas. Por ejemplo, haces un atole para las mujeres que tienen muy poca leche... Si están amamantando las ayuda a generar más. Ese es el efecto (Catalina, 45 años, México).
\end{abstract}

La variedad de plantas mencionadas en las preparaciones incluyó especies de amplia distribución, algunas de ellas exclusivamente medicinales (manzanilla, tila, cola de caballo, valeriana, ricino, quina) o empleadas como ingredientes culinarios (orégano, romero, jengibre, limón, cebolla, ajo). La principal forma de consumo referenciada fue mediante infusiones, aunque también se mencionaron aceites bebibles y para masaje, compresas, emplastos, enemas y baños de asiento. En la mayoría de los casos se trata de productos habituales en España, fáciles de conseguir, o de uso doméstico frecuente. En el caso específico de ciertas especies autóctonas americanas, algunas entrevistadas mencionaron obtenerlas en comercios especializados (herbolarios, tiendas de productos latinos) o mediante viajes al país de origen (propios o de personas conocidas):

Cuando no las encuentro aquí, una vez que voy a mi país traigo todas mis raíces de allá y las mantengo aquí. Con eso hago todos mis remedios naturales. Entonces lo que pueda traer yo, lo traigo. Lo que pasa también es que a veces viaja un amigo y me trae, o yo le traigo a los amigos... Y así nos mantenemos. Hay también algunos locutorios latinos que tienen productos muy buenos, que vienen muy frescos (Esther, 47 años, República Dominicana).

Aparte de los recursos de la fitomedicina casera fueron referidas, en determinados casos, acciones rituales de raigambre católica como rezos, conjuros, curas de palabra, baños de agua bendita, u otras prácticas como las limpias, friegas, tiradas de cuero o pellejo. Esto fue indicado en relación con ciertos padecimientos tradicionales o populares de América Latina, como el mal de ojo, el empacho, la pasada de calor o los culucos en el caso de los bebés.

El empacho, bueno, tienes que sobarte. Tienen que darte una sobada porque son como unas pelotas que tiene en el estómago... (Valentina, 50 ańos, Honduras).

Mi hermana me curaba a mis hijas de ojo. Les pasaba el huevo, después tenía que venir alguien y prestarle una camisa sudada... luego mascar guaro con ruda y rociar al bebé. Al final el huevo lo echan en un vaso y lo ponen debajo de donde el bebé duerme. Si se le forma el ojito al huevo, entonces ahí se confirmaba que era ojo (Rita, 39 años, Honduras). 
La incidencia de este tipo de malestares, no obstante, se reconoció menor en España. Comparativamente, la mayor parte de las acciones de autotratamiento reportadas por las mujeres tras el desplazamiento se destinó a resolver aquellas problemáticas comunes previamente mencionadas.

Finalmente, durante las entrevistas se llegó a señalar la utilización ocasional de medicamentos de patente en el itinerario de autoatención. En estas circunstancias suele ser también la mujer la encargada de seleccionar el producto, la dosis y la duración del tratamiento, basándose en recomendaciones de personas cercanas o en experiencias previas. La preferencia por las terapias denominadas "naturales" (fitomedicina casera) fue resaltada rotundamente por casi todas las participantes, aludiendo a los posibles efectos secundarios del consumo de medicinas industriales.

\section{"En nuestras casas y en las de otros": mujeres y el ejercicio del cuidado}

Además de la realización de prácticas curativas, un segundo conjunto de actividades vincula a las mujeres con el ámbito de la salud: las tareas de cuidado y responsabilización respecto de los miembros de la familia o de terceros. Se ha mencionado anteriormente que ciertas actividades cotidianas — habitualmente relegadas a manos femeninas- de limpieza, nutrición, educación y crianza, contribuyen en el establecimiento de contextos propiciadores de la salud o saludables.

Estas actividades de asistencia pueden llegar a constituirse, en muchas ocasiones, una forma de sustento económico. En Espańa, el cuidado de niños pequeños, personas enfermas o ancianos con distintos grados de morbilidad representa una de las principales alternativas de empleo accesibles a las mujeres inmigrantes (CRIADO, 2001; COMAS D'ARGEMIR, 2015). La alta demanda de servicios orientados a las familias se vuelve un factor atractor importante para el desplazamiento, especialmente en el caso de las personas latinoamericanas quienes cuentan con la ventaja de hablar el mismo idioma que la sociedad de destino (MARTÍNEZ-BUJÁN, 2010). Como se mencionó anteriormente, entre las diecisiete mujeres entrevistadas, siete se encontraban trabajando como cuidadoras de adultos mayores al momento de la investigación; y de las ocho participantes desempleadas, cinco habían trabajado en ese rubro en algún momento desde su llegada. Las tareas como empleada cuidadora incluyen el aseo personal y acompañamiento de la persona atendida, el control y dispensación de medicamentos, el chequeo de funciones corporales y la vigilancia de síntomas, la 
solicitud de consultas y el acompañamiento en caso de visita al médico. También suele requerirse la realización de tareas adicionales del hogar como limpieza y cocina.

Concretamente en el caso de las mujeres de la región Caribe, fue posible identificar el cuidado a terceros como un espacio adicional en el que emplear la medicina casera. Aparte de realizar las tareas habitualmente solicitadas, algunas de ellas aplican sus conocimientos terapéuticos para aliviar malestares comunes en los individuos asistidos:

A veces ayudo fuera de casa con estas recetas. Pero cosas naturales, un té. $\mathrm{O}$ eso del jengibre. La señora donde yo trabajo estaba con tos y le dije del jengibre (Alejandra, 50 años, Venezuela).

Aquí he cuidado mucha gente mayor... Ahora estoy cuidando un señor de 97 años y tenía muchos problemas de estreńimiento. Le di medio vaso de aceite de girasol por la noche y al día siguiente estaba aliviado. Son cosas que me agradecen mucho. Le hago mis remedios a la gente que yo cuido (Isabel, 41 ańos, Honduras).

Estas acciones no suelen ser explícitamente solicitadas por sus empleadores, sino que son las propias mujeres quienes las incorporan dentro de sus estrategias particulares de trabajo. En otros casos también suelen actuar activamente sobre el bienestar emocional de las personas dependientes, al reconocer en ellas ciertas necesidades afectivas:

Las viejitas son muy dadas a que las abracen y las apapachen. Pasan muchas necesidades de eso. Nos buscan para darles esa atención (Beatriz, 34 años, El Salvador).

Que yo llegara a trabajar le levantó mucho el ánimo a la señora. Estaba deprimida... Quería alguien que la cuidara más a ella que a la casa (Eva, 51 años, Venezuela)

Existe una carga diferencial amplia en el trabajo de cuidado para hombres y mujeres, y aunque no representa una exclusividad — también hay cuidadores masculinos - la actividad se encuentra culturalmente feminizada (ESTEBAN, 2006; VALDERRAMA-PONCE, 2006). Algunos testimonios vincularon la asistencia y atención doméstica con las tareas habituales designadas al género, explicándolas desde la configuración del rol materno hasta llegar a la propia cultura latinoamericana. Esto forma parte, de acuerdo con Scavino-Solari (2017), de los mismos mecanismos discursivos mencionados anteriormente con las prácticas curativas, en los que las mujeres reproducen el orden social establecido:

Me imagino que las mujeres somos más responsables de cuidar por la cultura nuestra, donde es la mamá quien pasa más pendiente de sus hijos. En otros países supongo que es igual. El hombre se encarga de trabajar supuestamente, y una desde casa. Pero a veces 
a una le toca también trabajar afuera de la casa y siempre le toca igual tener que hacer esas cosas. Ellos también pueden, pero no lo hacen porque no quieren. Son machistas (Rita, 39 años, Honduras).

De la salud familiar y ese tipo de cosas se ocupa más la mujer. Sí, creo que más la mujer. Sí. La mujer es más activa siempre... Lleva todo... Es múltiple... (Eva, 51 ańos, Venezuela).

$\mathrm{Al}$ igual que como ocurre con la asistencia intrafamiliar, la relegación femenina a este ámbito laboral es, según Gregorio-Gil (2017), producto de demarcaciones históricas de género. La percepción, la empatía y el afecto son vistos como caracteres esencialmente femeninos, necesarios en las tareas de asistencia. Asimismo, la maternidad como facultad biológica distintiva es una base fuerte sobre la que se funda dicha normalización.

Respecto a la contratación de personal doméstico extranjero se ha documentado ampliamente la preferencia por las cuidadoras latinoamericanas respecto de otras nacionalidades (MORÉ, 2018; DÍAZ-GORFINKEL; MARTÍNEZ-BUJÁN, 2018). Dentro de la sociedad española es frecuente la apelación a imágenes y construcciones estereotípicas que vinculan de forma específica a las latinas con una mayor "idoneidad" para el cuidado. Los discursos enumeran cualidades como la calidez, dulzura y sensibilidad, así como también asocian la idea de una fuerte educación familiar orientada a la domesticidad y el servilismo. Estas estrategias forman parte también del aparato legitimador que sostiene la división genérica del trabajo y justifica la posición de estas mujeres en dicho sistema laboral y estructura social (BERMÚDEZ-QUINTANA, 2006; HONDAGNEU-SOTELO, ESTRADA, RAMÍREZ, 2011). En repetidas ocasiones, las participantes reconocieron y reprodujeron estos mensajes, considerando que las favorecen al momento de conseguir empleo en el sector. No obstante, algunas de ellas se mostraron frustradas ante los posibles reduccionismos y la frecuente desestimación de otros logros y capacidades personales:

Yo tengo más cosas que ofrecer que simplemente cambiar pañales. Tengo un título, he hecho mis estudios en Administración. No puede ser que aquí sólo nos ofrezcan eso. Estamos igual o mejor capacitados que las personas españolas (Lydia, 35 ańos, Honduras).

Esto fue así sobre todo para las mujeres jóvenes, quienes no esperaban que la atención doméstica de terceros fuera casi la única alternativa de inserción laboral tras su llegada. 


\section{Consideraciones finales}

La participación mayoritaria de las mujeres en el cuidado y la curación evidencia una compleja configuración social y cultural que construye significados y entreteje relaciones de poder, clase y género. Dicha configuración, producto de una historia determinada, encuentra en la actualidad formas de manifestarse y perpetuarse (RAMOS-ESCANDÓN, 1997). Tal y como refiere Foucault, "los códigos fundamentales de una cultura - los que rigen su lenguaje, sus esquemas perceptivos, sus cambios, sus técnicas, sus valores, la jerarquía de sus prácticas - fijan de antemano para cada hombre los órdenes empíricos con los cuales tendrá algo que ver y dentro de los que se reconocerá...” (1978: 5). Resulta valioso continuar analizando estos elementos desde una perspectiva interseccional como la propuesta por Hofman (2010), para echar luz sobre las estructuras de diferenciación y el modo en que se interpolan en la experiencia de los distintos sujetos.

El caso concreto de las migrantes latinoamericanas en España nos muestra una realidad paradójica respecto de la valoración social del género en el contexto migratorio. Por un lado, la trasmisión intergeneracional de conocimientos curativos y la perpetuación de la tradición cultural nativa en manos femeninas supone para las mujeres una forma de distinción y empoderamiento, al permitirles reconocerse portadoras y dispensadoras de un saber importante para la comunidad. Por otra parte, el relegamiento a las tareas domésticas y de cuidados como forma de subsistencia da cuenta de las estructuras de desigualdad y marginación que se erigen y sustentan en la conjunción del género y el origen étnico. Las mismas actividades propiciadoras de salud que son valoradas por sus practicantes al interior del grupo, pasan a ser instrumentadas por terceros - en este caso la sociedad de destino convirtiéndolas en símbolos de subordinación y confinamiento. ${ }^{1}$

\section{Referencias}

ASSAE. Asociación de Antropología del Estado Español. Orientaciones deontológicas para la práctica de la Antropología profesional. 2014. Disponible en: http://asaee-antropologia.org/asaee/ codigo-deontologico/ [Revisado el 10/10/2016]

AUBARELL, G. Una propuesta de recorrido bibliográfico por las migraciones femeninas en España. Papers, n. 60, p. 391-413, 2000. 
BAEZA, B.; AIZENBERG, L; BARRÍA, C. Cultura y salud migratoria: miradas comparativas entre profesionales sanitarios y mujeres migrantes bolivianas. Si somos americanos, v. 19, n. 1, p. 43-66, 2019.

BARRAGÁN-SOLÍS, A. La práctica de la autoatención por fitoterapia en un grupo de familias mexicanas. Archivos en Medicina Familiar, v. 8, n. 3, p. 155-162, 2006.

BARTOLI, P. ¿Esperando al doctor? Reflexiones sobre una investigación de antropología médica en México. Revista de Antropología Social, v. 14, p. 71-100, 2005.

BERMÚDEZ-QUINTANA, M. Mujeres inmigrantes y salud, testimonios colombianos. Valencia: Universitat de València, 2006

CASTLES, S; MILLER, M. The age of migration: international population movements in the modern world. Nueva York: The Guilford Press, 1998.

COLECTIVO IOÉ. La sociedad española y la inmigración extranjera. Papeles de economía española, n. 98, p. 16-31, 2003.

COMAS D’ARGEMIR, D. Los cuidados de larga duración y el cuarto pilar del sistema de bienestar. Revista de Antropología Social, v. 24, p. 375-404, 2015.

CRIADO, M. La línea quebrada. Historias de vida de migrantes. Madrid: Consejo económico y social (CES), 2001.

DÍAZ-GORFINKIEL, M.; MARTÍNEZ-BUJÁN, R. Mujeres migrantes y trabajos de cuidados: transformaciones del sector doméstico en España. Panorama Social, n. 27, 105-118, 2018.

DYCK, I.; DOSSA, P. Place, health and home: Gender and migration in the constitution of healthy space. Health and Place, v. 13, n. 3, p. 691-701, 2007.

ESTEBAN, M. El Estudio de la Salud y el Género: Las Ventajas de un Enfoque Antropológico y Feminista. Salud Colectiva, v. 2, n. 1, p. 9-20, 2006.

FOUCAULT, M. Las palabras y las cosas. México D.F.: Siglo XXI Editores, 1978.

GENTIL, I. Salud y mujeres inmigrantes latinoamericanas. Autoestima y resiliencia. Index de Enfermeria, v. 18, n. 4, p. 229-233, 2009.

GREGORIO-GIL, C. ¿Por qué hablar de cuidados cuando hablamos de migraciones transnacionales? Quaderns-e Instituto Català d'Antropologia , v. 22, n. 2, p. 49-64, 2017.

GUEST, G.; MACQUEEN, K.; NAMEY, E. Applied thematic analysis. Thousand Oaks: SAGE Publications, 2012.

GUIJÓN-SÁNCHEZ, M. T.; JIMÉNEZ-RODRIGO, M. L.; MARTÍNEZ-MORANTE, E. Más allá de la diferencia, tras el cristal de la diversidad. La "inmigración” en la literatura biomédica en España. En: FERNÁNDEZ-JUÁREZ, G. (Coord.), Salud e interculturalidad en América Latina. Antropologia de la salud y critica intercultural. Quito: Ediciones Abya Yala, 2006, p. 115-132. 
HAMUI, L. Las narrativas del padecer: una ventana a la realidad social. Cuicuilco, v. 18, n. 52, p. 51-70, 2011.

HERNÁNDEZ-TEZOQUIPA, I.; ARENAS-MONREAL, M.; VALDE-SANTIAGO, R. El cuidado a la salud en el ámbito doméstico: interacción social y vida cotidiana. Revista de Saúde Pública; v. 35, n. 5, p. 443-450, 2001.

HERRERA-SANTI, P. Rol de género y funcionamiento familiar. Revista cubana de Medicina General Integral; v. 16, n. 6, p. 568-573, 2000.

HOFMAN, N. Understanding women's work through the confluence of gender, race and social class. Cultural dynamics; v. 22, n. 3, p. 179-195, 2010.

HONDAGNEU-SOTELO, P.; ESTRADA, E.; RAMÍREZ, H. Más allá de la domesticidad. Un análisis de género de los trabajos de los inmigrantes en el sector informal. Papers, v. 96, n.3, p. 805-824, 2011.

IDOYAGA-MOLINA, A. La clasificación de las medicinas, la atención de la salud y la articulación de factores culturales, sociales, económicos y étnicos. Itinerarios terapéuticos en contextos pluriculturales y multiétnicos de Argentina. En: IDOYAGA-MOLINA, A. (ed.) Los caminos terapéuticos y los rostros de la diversidad. Tomo II. Buenos Aires: Editorial CAEA-IUNA, 2007, p. 5-65.

JORAND, B. Formas de transformación del conocimiento de la medicina tradicional en los pueblos nahuas del municipio de Hueyapán, Sierra Norte de Puebla. Cuicuilco; n. 44, p. 181-196, 2008.

JUÁREZ, F. El concepto de salud: una explicación sobre su unicidad, multiplicidad y los modelos de salud. International Journal of Psychological Research, v. 4, n. 1, p. 70-79, 2011.

KLEINMAN, A. Concepts and a model for the comparison of medical systems as cultural systems. Social Science and Medicine; v. 12, p. 85-93, 1978.

Basic Books, 1988.

LLOSADA-GISTAU, J. et al. Acceso y uso de los servicios sanitarios por parte de los pacientes inmigrantes: la voz de los profesionales. Atención Primaria, v. 44, n. 2, p. 82-88, 2012.

MARTÍNEZ-BUJÁN, R. Servicio doméstico y trabajo de cuidados. Hacia la privatización del cuidado familiar. Alternativas. Cuadernos de Trabajo Social, n. 17, p. 157-179, 2010.

La reorganización de los cuidados familiares en un contexto de migración internacional. Cuadernos de Relaciones Laborales, v. 29, n. 1, p. 93-123, 2011.

MEDINA-CÁRDENAS, E. Autoatención doméstica de la salud. III Congreso Chileno de Antropología. Colegio de Antropólogos de Chile A. G, Temuco. 1998. Disponible en: https://www. aacademica.org/iii.congreso.chileno.de.antropologia/90.pdf [Revisado el 20/01/19] 
MENDELSON, C. The roles of contemporary Mexican American women in domestic health work. Public Health Nursing, v. 20, n. 2, p. 95-103, 2003.

MENÉNDEZ, E. Familia, participación social y proceso salud/enfermedad/atención. Acotaciones desde la perspectiva de la antropología médica. En: MERCADO, F. et al. (coord.) Familia, salud y sociedad: Experiencias de investigación en México. México: Universidad de Guadalajara, IISP, CIESAS, 1993, p. 130-162.

MENJÍVAR, C. The ties that heal: Guatemalan immigrant women's networks and medical treatment. International Migration Review, v. 36, n. 2, p. 437-466, 2002.

MESSIAS, D. K. H. Transnational health resources, practices and perspectives: Brazilian immigrant women's narratives. Journal of Immigrant Health, v. 4, n. 4, p. 183-200, 2002.

MIELES-BARRERA, M.; TONON, G.; ALVARADO-SALGADO, S. Investigación cualitativa: el análisis temático para el tratamiento de la información desde el enfoque de la fenomenología social. Universitas Humanistica; n. 74, p. 195-225, 2012.

MORÉ, P. Los límites del cuidado. Organización y asistencia a domicilio para personas mayores. Revista Internacional de Sociología, v. 76, n. 1, e086, 2018. Disponible en: https://doi. org/10.3989/ris.2018.76.1.16.25 [Revisado el 30/04/19]

MORGAN-CONSOLI, M.; UNZUETA, E. Female Mexican immigrants in the United States: Cultural knowledge and healing. Women \& Therapy; v. 41, n. 1-2, p. 165-179, 2018.

ORGANIZACIÓN PANAMERICANA DE LA SALUD. Sistemas de salud tradicional en América Latina y el Caribe. Washington: Organización Panamericana de la Salud - División de Desarrollo de Sistemas y Servicios de Salud. 1999.

ORTNER, S. ¿Es la mujer con respecto al hombre lo que la naturaleza con respecto a la cultura? En: HARRIS, O.; YOUNG, K. (eds.) Antropología y feminismo. Barcelona: Anagrama, 1979, p. 109-131.

OSO, L. Mujeres migrantes en España. Bastiones de la resistencia tras la crisis económica. Anuario CIDOB de la inmigración, p. 130-143, 2018. Disponible en: https:/www.raco.cat/index.php/ AnuarioCIDOBInmigracion/article/view/10.24241-AnuarioCIDOBInmi.2018.130/435066 [Revisado el 28/04/19]

OSORIO-CARRANZA, R. Entender y atender la enfermedad. Los saberes maternos frente a los padecimientos infantiles. México: CIESAS, 2001.

La trayectoria de atención de las enfermedades infantiles. Una puesta en escena de las representaciones y prácticas sociales. En: ESTEBAN, M. L. (ed.) Introducción a la Antropología de la salud. Aplicaciones teóricas y prácticas. Bilbao: OSALDE, Asociación por el derecho a la salud, p. 151-175, 2007. 
PEZO-SILVA, M.; DE SOUZA-PRAÇA, N.; COSTA-STEFANELLI, M. La mujer responsable de la salud de la familia. Constatando la universalidad cultural del cuidado. Index de Enfermeria, v. 13, n. 46, p. 13-17, 2004.

RAMOS-ESCANDÓN, C. El concepto de "género" y su utilidad para el análisis histórico. La Aljaba, segunda época; v. 2, p. 13-32, 1997.

RÍO-BENITO, M. J. et al. Las mujeres migrantes en Sevilla: salud y condiciones de trabajo. Una revisión desde la medicina social. REIS: Revista Española de Investigaciones Sociológicas, n. 104, p. 123-144, 2003.

REGIDOR, E. et al. La utilización de los servicios sanitarios por la población migrante en España. Gaceta Sanitaria, v. 23, n. 1, p. 4-11, 2009.

SÁNCHEZ-BIRKHEAD et al. Navigating a new health culture: experiences of immigrant Hispanic women. Journal of Immigrant and Minority Health, n. 13, p. 1168-1174, 2011.

SCAVINO-SOLARI, S. Cuidados y subjetivación de género. Un análisis de discurso de las mujeres que constituyen hogares monoparentales con hijos pequeños. Psicología, Conocimiento y Sociedad, v. 7, n. 1, p. 141-168, 2017.

SILES-GONZÁLEZ, J.; SOLANO-RUIZ, C. Estructuras sociales, división sexual del trabajo y enfoques metodológicos. La estructura familiar y la función socio-sanitaria de la mujer. Investigación y Educación en Enfermería, v. 25, n. 1, p. 66-73, 2007.

UNDA, R.; ALVARADO, S. Feminización de la migración y papel de las mujeres en el hecho migratorio. Revista Latinoamericana de Ciencias Sociales, Niñez y Juventud, v. 10, n. 1, p. 593610, 2012.

VALDERRAMA-PONCE, M. El cuidado, ¿Una tarea de mujeres? Vasconia, n. 35, p. 373$385,2006$.

VAQUIRO-RODRÍGUEZ, S.; STIEPOVICH-BERTONI, J. Cuidado informal, un reto asumido por la mujer. Ciencia y enfermería, v. 16, n. 2, p. 9-16, 2010.

VELASCO, L. El regreso de la comunidad: migración indígena y agentes étnicos. Los mixtecas en la frontera México-Estados Unidos. Tijuana: El Colegio de México. El Colegio de la Frontera Norte, 2002.

VOLPATO, G.; GODÍNEZ, D.; BEYRA, A. Migration and ethnobotanical practices: The case of tifey among Haitian immigrants in Cuba. Human Ecology, v. 37, n. 1, p. 43-53, 2009.

WALDSTEIN, A. Popular medicine and self-care in a Mexican migrant community: toward an explanation of an epidemiological paradox. Medical Anthropology, v. 29, n. 1, p. 71-107, 2010. WILKINSON, D. Traditional Medicine in American Families: reliance on the wisdom of elders. Marriage \& Family Review; n. 11, p. 65-76, 1987. 
ZAPPAROLI-ZECCA, M. Mujeres y prácticas curativas tradicionales. Revista Reflexiones; v. 91, n. 2, p. 107-119, 2012.

\section{Nota}

${ }^{1}$ M. Lamarque y L. M. Gutiérrez realizaron el diseño de la investigación, revisión bibliográfica, trabajo de campo, análisis de resultados y redacción de artículo. 


\section{Abstract}

Healing inside, caring outside: Latin American women and therapeutic practices in the transnational migration context

The article attempts to reflect on the relationship between Latin American immigrant women and therapeutic practices, according to the roles designated or assumed by them in healthcare processes. An ethnographic research was carried out with Caribbean women settled in Spain, in order to inquire about certain curative and care activities exercised by them, both inside and outside their homes. In addition to the descriptive component, we worked specifically with the narratives and meanings elaborated around these occupations. Their analysis allowed us to identify conflicting facets in the development of female identity and migratory experience. Being a caregiver as their main job alternative evidence the inequality of opportunities and social impact areas accessible to Latin women; at the same time, they reproduce essentialist schemes, which naturalize the division of labour according to ethnic and gender "qualities". On the other hand, the management of specific knowledge about healing, treatment and assistance allow women to possess a distinctive and potentially empowering role within the community as continuators of certain traditional knowledge.

Keywords: migration; popular medicine; therapeutical practices; care; gender roles. 


\section{Resumo}

Curando dentro, cuidando fora: mulheres

latino-americanas e práticas terapêuticas no

contexto migratório transnacional

O presente trabalho procura refletir sobre a relação

que se constrói entre as mulheres latinas migrantes

e as práticas terapêuticas, de acordo com os papéis

designados ou assumidos por elas nos processos de

saúde/doença/cuidado. Uma pesquisa etnográfica

foi realizada com mulheres caribenhas instaladas na

Espanha, a fim de indagar sobre certas atividades

curativas e cuidados exercidos por elas, tanto

dentro como fora de casa. Além do componente

descritivo, trabalhamos com narrativas e significados

específicos elaborados em torno dessas ocupaçóes.

Sua análise permitiu identificar facetas conflitantes

no desenvolvimento da identidade feminina e da

experiência de migração. As atividades de cuidado como principal alternativa de trabalho evidenciam a

desigualdade de oportunidades e áreas de impacto social acessíveis aos latino-americanos; ao mesmo tempo,

refletem mecanismos de reprodução de esquemas essencialistas, que naturalizam a divisão do trabalho de acordo com "qualidades" étnicas e de gênero. Por outro lado, a gestão de conhecimentos específicos sobre cura, tratamento e assistência supóe para as mulheres a posse de um papel distintivo e potencialmente empoderador dentro da comunidade como continuadores de certos conhecimentos tradicionais.

> Palavras-chave: migração; medicina popular; práticas terapêuticas; cuidados; papéis de gênero. 